\title{
Statyba
}

\section{METHODS OF SYNTHESIS OF TECHNICAL AND ORGANISATIONAL-TECHNOLOGICAL SOLUTIONS IN CONSTRUCTION}

\section{E. K. Zavadskas \& L. Ustinovičius}

To cite this article: E. K. Zavadskas \& L. Ustinovičius (1999) METHODS OF SYNTHESIS OF TECHNICAL AND ORGANISATIONAL-TECHNOLOGICAL SOLUTIONS IN CONSTRUCTION, Statyba, 5:4, 285-289, DOI: 10.1080/13921525.1999.10531476

To link to this article: https://doi.org/10.1080/13921525.1999.10531476

Published online: 26 Jul 2012.

Submit your article to this journal $\llbracket$

Џ Article views: 45 


\section{SYNTHESEMETHODEN DER TECHNISCHEN UND ORGANISATORISCH- TECHNOLOGISCHEN LÖSUNGEN IM BAUWESEN}

\section{E. K. Zavadskas, L. Ustinovičius}

\section{Einführung}

In der Baupraxis sind oft die Notwendigkeit der Lösung der Aufgaben der Mehrzielselektierung (MS) bei der Synthese der baulichen Variante zu treffen, die aus mehreren miteinander verbundenen technischen und organisatorisch-technologischen Lösungen besteht.

\section{Hauptaufgaben der Synthese}

Die Erfahrung der technologischen Lösung der Bauproduktion zeigt, daß die Hauptaufgaben der Synthese auf folgender Weise klassifiziert werden können:

die Ermittlung der Bevorzugung der Lösungsvarianten, wenn die Teillösungen in den einzelnen Etappen nach getrennten Gruppen der Kennziffern der Effektivität (KE) getroffen werden, die sich durch die Art der Kennziffern der Effektivität und ihre Parameter unterscheiden;

die Ermittlung der Bevorzugung der Lösungsvarianten, wenn die Teil-(Etappen)-lösungen nach den Gruppen der Effektivitätskennziffern getroffen werden können, die für alle Teil-(Etappen-)-lösungen gleich sind.

\section{Die Ermittlung der Bevorzugung der}

\section{Lösungsvarianten, wenn die getrennten Gruppen der} Kennziffern der Effektivität getroffen werden

Dabei kann eine jede Teil-(Etappen)-lösung aus zwei oder einer größeren Anzahl der möglichen Varianten getroffen werden. Die Lösungen aus zwei möglichen Varianten können nach der Methodik des Netzwerkdiagrammes der Entscheidung (NE) getroffen werden. Wenn es eine Notwendigkeit ist, die Synthese der Lösung bei der Anwendung mehr als zwei Varianten in jeder Lösungsetappe durchzuführen, so sind zwei Vorgehensweisen möglich. Bei der ersten Vorgehensweise wird die Auswahl von zwei Varianten vorgenommen, dabei werden in jeder Etappe zwei am meisten bevorzugte Varian- ten genommen. Vor und nach der Variantenauswahl sind die Sonderrechnungen vorzunehmen die Wahrscheinlichkeitsziffern zu ermitteln u.s.w. Dieses Verfahren ist für die Lösung der Aufgaben bei der Einschätzung der einzelnen Etappenlösungen nach verschiedenen Effektivitätskennziffern geeignet. Die Aufgabe wird nach dem folgenden Algorithmus $\mathrm{Cl}$ (Bild 1) gelöst.

Nehmen wir an, daß es $k$ Etappen der Lösung $(k=\overline{1, N})$ gibt. In einer jeden Etappe $k$ wird die Auswahl aus $i$ Varianten $(i=\overline{1, m})$ nach $j$ der Effektivitätskennziffern vorgenommen $(j=\overline{1, n})$. In der ersten Etappe wird die Matrix der Verbindungen der Etappenlösungen aufgestellt. Durch die Methode der Entropie [1] werden die Kennzahlen der Wichtigkeit der Effektivitätskennziffern in einer jeden Etappe $k$ mit der nachfolgenden Bestimmung der Bevorzugung der Varianten nach dem Kriterium des durchschnittsgewichteten Erfolges der getroffenen Lösung (dEgL) nach der Formel (l):

$$
\begin{gathered}
K=a_{i}=\left\{a_{i} / A_{i} \in A_{i} \cap \max _{i} \frac{1}{n} \sum_{j=1}^{n} q_{i} \overline{x_{i j}} \cap\right. \\
\left.\cap \max _{i} \frac{1}{n} \sum_{j=1}^{n} q_{i} x_{i j}\right\}, \\
\forall i j ; \quad i=\overline{1, m} ; \quad j=\overline{1, n} ;
\end{gathered}
$$

ermittelt, wo $x_{i j}$ der Wert der $j$ Kennnziffer der Variante $i$ ist.

Aus der erhaltenen Reihe der Bevorzugung werden zwei erste Varianten angenommen, für die die Berechnung wiederholt wird. Auf solche Weise wird die Wichtigkeit der Varianten $w_{i k}$ aus der Bedingung $\sum_{i=1}^{2} w_{i k}=1$ ermittelt. Die Reihe der Bevorzugung der zu synthesierenden Varianten (Zweige) wird auf Grund der Regelung nach dem Kriterium gebildet, das folgenderweise ermittelt wird:

$$
\Omega_{s}=\max \prod_{\substack{i=1 \\ k=1}}^{m, N} w_{i}, k=\overline{1, N}, i=\overline{1, m} ; s=\overline{1, d} .
$$

Ausgehend aus der Bedingung, da 3 die beste Variante $\left(a_{s}, s=\overline{1, d}\right)$ die ist, deren Wert $\Omega_{s}$ maximal ist, 


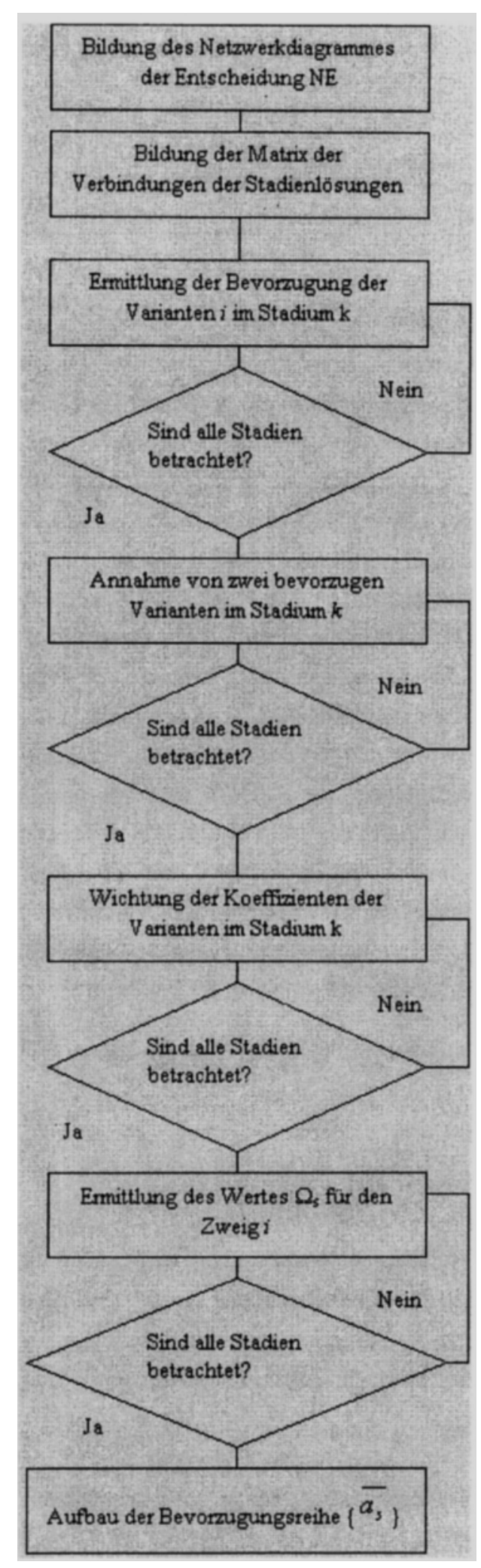

Bild 1. Block-Schema des Algorythmus $\mathrm{Cl}$ der Synthese der technischen und organisatorisch-technologischen Lösungen

Fig 1. Scheme of algorithm $\mathrm{Cl}$ for technical and organisational - technological solutions synthesis wird auf Basis schon bekannten Werte $\Omega_{s}$ die Reihe der Bevorzugung der Varianten gebildet:

$$
\left\{a_{s}\right\}=\left\{a_{1}>a_{3}>a_{4}>\ldots>a_{s}>\ldots>a_{d}\right\} \text {. }
$$

wo s die Anzahl der Zweige der NE ist.

Bei der Notwendigkeit der Synthese der Lösungen bei der Anwendung mehr als zwei Varianten in einer jeden Etappe kann die Lösung auch nach dem unten dargebotenem Algorithmus C2 (Bild 2) durchgeführt werden.

Nehmen wir an, daß für die Realisierung der Projektlösung die Auswahl $k(k=\overline{1, N})$ der unterschiedlichen nach der Funktionsbestimmung der Konstruktionen notwendig ist. Eine jede konstruktive Lösung kann aus $m$ möglichen Varianten ausgewält werden. Dabei kann die Anzahl der Varianten $m$ verschieden für die Konstruktionen der verschiedenartigen funktionalen Bestimmung sein, die auch dazu noch mit verschiedenen Effektivitätskennziffern kenngezeichnet werden. Die Lösung einer solchen Aufgabe wird folgenderweise vorgenommen es wird die Matrix der Verbindungen der Lösungsvarianten gebildet weiterhin wird durch die Entropiemethode die Effektivitätskennziffern einer jeden Etappe $k$ gewichtet. Die zu ermittlenden Koeffizienten des Gewichtes der Effektivitätskennziffern müssen die Bedingung $\sum_{j=1}^{n} q_{j}=1$ erfüllen (wo $j$ die Anzahl der Effektivitätskennziffern in jeder Etappe $k$ ist). Durch Anwendung der Kriterien des durchschnittsgewichte Erfolges der zu treffenden Lösung ( $\mathrm{dEgL}$ ) werden die Koeffizienten der Wichtigkeit der zu vergleichenden Varianten in jeder Etappe der zu treffenden Lösung $k$ ermittelt. Dabei wird die Bedingung $\sum_{j=1}^{m} w_{i}=1$ erfüllt.

Folglich wird von der Anzahl $i(i=\overline{1, m})$ der zu vergleichenden Varianten in jeder Etappe auch der absolute Wert der ermittelten Koeffizienten der Wichtigkeit der Effektivitätskennziffern abhängen. Je weniger gibt es die $z u$ vergleichenden Varianten $i$, um so größere Werte werden die Werte $w_{i}$ haben. Auf solche Weise ist die Regelung des Wertes $w_{i}$ in der Abhängigkeit von der Anzahl der $\mathrm{zu}$ vergleichenden Varianten so notwendig, damit diese Werte richtig bei der Auswahl der endgültigen am meisten bevorzugten Variante berücksichtigt waren. Für diese Zwecke werden die Proportionen gelöst, in dem als 


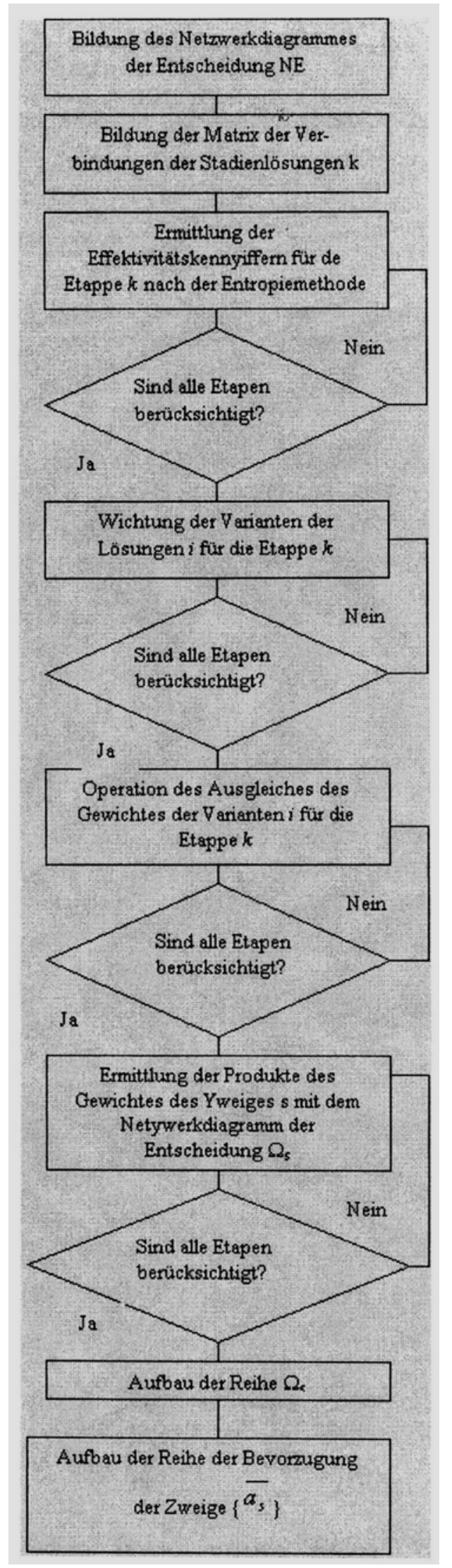

Bild 2. Block-Schema des Algorythmus C2 der Synthese der technischen und organisatorisch-technologischen Lösungen

Fig 2. Scheme of algorithm $\mathrm{C} 2$ for technical and organisational - technological solutions synthesis
Ausgang zwei Varianten mit $\sum W_{i}=1$ genommen werden. Dann ist bei vier Vergleichsvarianten $\sum_{j=1}^{m} w_{i}=2$ und bei fünf entsprechend 2,5 u.s.w. Die durch die Anwendung der Entropiemethode erhaltenen Werte der Koeffizienten der Wichtigkeit der Varianten $i$ sind mit den entsprechenden Koeffizienten zu multiplizieren, die die Anzahl der zu analysierenden Varianten in derb einzelnen Etappen Berücksichtigen. Die Werte der Koeffizienten werden nach der elementären Formel bestimmt:

$$
h_{k}=i_{k} / 2,
$$

wo $k$ die Anzahl der zu vergleichenden Varianten in der $m$ Etappe ist.

Auf solche Weise werden die linke und rechte Seiten des Ausdruckes $\sum_{i=1}^{m} w_{i}=1$ mit dem Wert des Koeffizienten $h_{k}$ multipliziert. Die erhaltenen Wertte $w_{i}=w_{i} h_{k}$ kennzeichnen die Wichtigkeit der einzelnen Lösungen in der bestimmen Etappe $k$.

Die endgültige Einschätzung wird durch die Multiplizierung aller $k$, die in verschiedenen Etappen entsprechen der Matrix der Verbindungen erhalten wurden der Werte $w_{k_{i}}$ durchgeführt. Nehmen wir an, daß die Entscheidung im Ergebnis der Einschätzung drei Etappenlösungen getroffen wird. Entsprechend der Matrix der Verbindungen wird der Zweig des Netzwerkprogrammes der Entscheidung 1-2-4 bewertet. Folglich wird zur Bewertung dieser Lösung das Produkt

$$
\Omega_{4}=w_{11} w_{22} w_{34},
$$

wo $\Omega_{4}$ die Einschätzung der Variante der vierten Lösung (des vierten Zweiges) ist. $w_{11}$ ist der Koeffizient der Wichtigkeit der ersten Variante der Lösung in der ersten Etappe der Entscheidungstreffung; $w_{22}$ ist der Koeffizient der Wichtigkeit der zweiten Variante der Lösung in der zweiten Etappe der Entscheidungstreffung; $w_{34}$ ist der Koeffizient der Wichtigkeit der vierten Variante der Lösung in der dritten Etappe der Entscheidungstreffung.

Die beste Variante der Synthese wird die, deren Größe $\Omega_{s}$ den maximalen Wert haben wird. Auf Grund der Werte $\Omega$ wird die Reihe der Bevorzugung der zu synthesierenden Varianten aufgebaut (3). 


\section{Die Ermittlung der Bevorzugung der Lösungs-} varianten, wenn alle Effektivitätskennziffern gleich sind

Bei zweiter Vorgehensweise werden in allen Etappen die Werte der preislichen, resourcelichen und zeitlichen Effektivitätskennziffern für alle Wege (Zweige) des Netzwerkprogrammes der Entscheidung (NE) summiert, die den zu erwartenden Ergebnissen der Realisation $a_{s} \in A$ (Bild3) entsprechen. Die Summierung der Werte der Effektivitätskennziffern wird nach dem Ausdruck

$$
x_{s j}=\sum_{\substack{i=1 \\ k=1}}^{m_{k}, N} x_{j k}, j=\overline{1, n_{k}}, k=\overline{1, N}, s=\overline{1, d}
$$

durchgeführt.

Auf solche Weise können bei der Anwendung der vorgeschlagenen Methode die Effektivitätskennziffern bewertet, die in allen Etappen der Entscheidungstreffung den Sinn der Summierung nicht verloren haben.

Auf Grund der Ergebnisse, die nach dem Ausdruck (6) erhalten sind, wird die Matrix der Lösungen $P=\left\lfloor x_{s j}\right\rfloor$ aufgestellt, d.h.

$$
P=\left[\begin{array}{cccccc}
x_{11} & x_{12} & \ldots & x_{1 j} & \ldots & x_{n_{k}} \\
x_{21} & x_{22} & \ldots & x_{2 j} & \ldots & x_{2 n_{k}} \\
\ldots & \ldots & \ldots & \ldots & \ldots & \ldots \\
\ldots & \ldots & \ldots & \ldots & \ldots & \ldots \\
x_{d 1} & x_{d 2} & \ldots & x_{d j} & \ldots & x_{d n_{k}}
\end{array}\right] .
$$

Weiterhin ist passend, die Lösung der am meisten bevorzugten Variante nach dem Kriterium der Nähe zum Idealen Punkt durchzuführen.

Die Beispiele der Anwendung dieses Kriteriums waren vielmals, in Deutschland veröffentlicht [2,3]. Außer diesem Kriterium können auch andere Methoden und Kriterien der Mehrzielselektierung angewandt werden [4].

Die Reihe der Bevorzugung der Zweige der Netzwerkprogramme der Entscheidung werden aufgestellt, indem man als die beste Variante mit der größten Wert des Kriteriums der Nähe zum idealen Punkt annimmt, d.h.

$$
\{a\}=\left\{a_{1}>a_{2}>\ldots>a_{s}>\ldots>a_{d}\right\} .
$$

\section{Schlußfolgerungen}

Weiterhin ist passend, die Lösung der am meisten bevorzugten Variante nach dem Kriterium der Nähe zum Idealen Punkt durchzuführen.

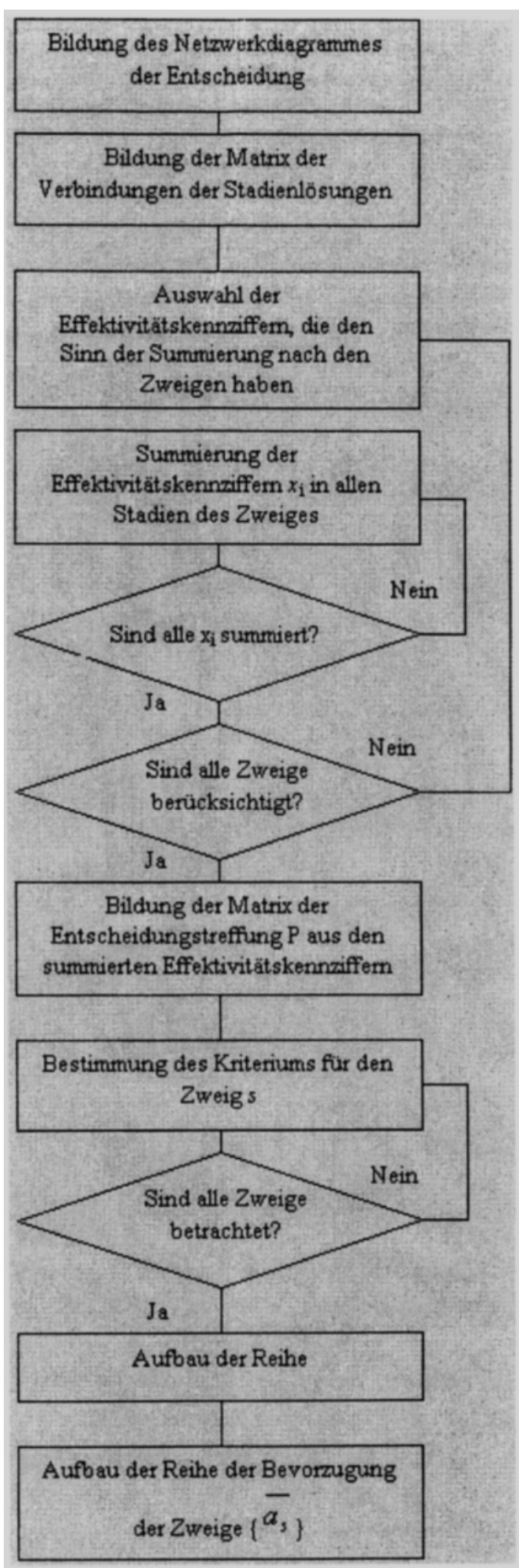

Bild 3. Block-Schema des Algorythmus C3 der Synthese der technischen und organisatorisch-technologischen Lösungen

Fig 3. Scheme of algorithm $\mathrm{C} 3$ for technical and organisational - technological solutions synthesis 
Die dargestellten Methoden wurden bei der Lösung einer Reihe der praktischen Aufgaben der Bauproduktion angewandt.

\section{Literatur}

1. Э. К. Завадскас. Комплексная оценка и выбор ресурсосберегаюших решений в строительстве. Вильнюс: Мокслас, 1987. 209 c.

2. A. Kaklauskas, L. Ruprecht, E. Zavadskas. Die Auswahl von Fundamenten für Dreigelenkrahmen einschiffiger Landwirtschaftsbauten nach mehreren Zielkriterien // Wissenschaftliche Zeitschrift der Hochschule für Architektur und Bauwesen Weimars. Weimar, 1988, S. 179-181

3. P. Altmann, F. Peldschus, E. Zavadskas. Neue Konzepte für die Dimensionierung von technologischen Linien für die Betonfertigteilproduktion // Wissenschaftliche Zeitschrift der TH. Leipzig, 1987. S. 135-142.

4. E. Zavadskas, F. Peldschus, A. Kaklauskas. Multiple Criteria Evaluation of Projects in Construction. Vilnius: Technika, 1994. $226 \mathrm{p}$.

Iteikta 19990717

\section{TECHNINIU IR ORGANIZACINIU-TECHNOLOGINIU STATYBOS SPRENDIMU SINTEZĖS METODAI}

\section{E. K. Zavadskas, L. Ustinovičius}

\section{S antrauka}

Statyboje dažnai tenka spręsti variantų sintezès daugiakriterinès atrankos uždavinius. Juos sudaro daug tarpusavyje susijusių techninių ir organizacinių-technologinių sprendinių.

Kiekvieno uždavinio etapo sprendimas gali būti sudarytas iš dviejų arba daugiau variantų.

Kai kiekvienas uždavinio etapas yra sudarytas iš dvieju variantu, uždavinị galima spręsti tinklinių diagramų būdu.

Kiekviename etape $k$ pagal $j$ efektyvumo kriterijus renkama iš $i$ variantų. Entropijos metodu nustatomi kiekvieno etapo efektyvumo rodiklių reikšmingumai. Variantų efektyvumas kiekviename etape nustatomas pagal kriteriju $k(1)$. Iš gautos prioritetų eilès atrenkami du pirmieji variantai, kurie pakartotinai perskaičiuojami pagal tą pačią metodika. Sintezuojamų variantų (šakų) atranka atliekama remiantis kriterijumi $\Omega_{\mathrm{s}}$ (2). Remiantis sąlyga, kad didesnè $\Omega_{\text {s }}$ reikšmè atitinka geriausią varianta, nustatoma variantų prioritetų seka (3).

Kai uždavinio etapuose yra daugiau negu du variantai, imamas antrasis algoritmas. Pradžioje sudaroma junginių matrica tarp atskirų etapų sprendinių. Entropijos būdu nustatomi kiekvieno etapo kriteriju reikšmingumai. Priklausomai nuo variantų skaičiaus bütina sureguliuoti reikšmingumų vertes. Entropijos büdu gauti reikšmingumai dauginami iš koeficiento, kuriuo ivvertinamas analizuojamų variantų skaičius (4).

Galutinis šakos ivertinimas gaunamas pagal 5 formulę. Geriausias sintezès variantas tas, kurio $\Omega_{\text {s }}$ reikšmè yra didžiausia.

Kai prioritetai nustatomi esant vienodoms efektyvumo rodiklių grupèms visuose etapuose, sumuojami visų tinklinès diagramos šakų visų etapų efektyvumo rodiklių reikšmingumai (3 algoritmas). Efektyvumo reikšmès sumuojamos pagal 6 formulę.

Variantų prioritetiškumo skaičiavimus galima atlikti ir atstumo iki idealaus taško metodu.

Minèti metodai buvo taikomi sprendžiant praktinius statybos uždavinius.

\section{METHODS OF SYNTHESIS OF TECHNICAL AND OR GANISATIONAL-TECHNOLOGICAL SOLUTIONS IN CONSTRUCTION}

\section{E. K. Zavadskas, L. Ustinovičius}

Sum mary

Quite frequently one has to solve variant synthesis multicriterial selection problems in construction. These problems consist of many technical-organisational solutions interacting with each other.

The solution of each problem can be made of two or more alternatives.

In each step $k$ according to $j$, the effectiveness criterion is selected from variants $i$. Using the entropy method the weights of effectiveness indices in each step are calculated. By the criterion $k(1)$ the effectiveness of variants in each step is determined. From the row of priorities obtained the first two variants are selected and they are repeatedly calculated by the same methods. The selection of variants (branches) synthesised is made by the criterion $\Omega_{s}(2)$. A priority row of variants is determined according to the rule that the greater value of $\Omega_{\mathrm{s}}$ means the best variant (3).

The second algorithm is used when there are more than two variants in the steps of problem. A joint matrix between separate steps of solutions is determined. Weights of criteria for each step are determined using the entropy method. It is necessary to regulate the values of importance according the number of variants. The weights obtained by the method of entropy are multiplied by the coefficient considering the number of alternatives analysed (4).

The final valuation of branch is obtained according to the formula No 5 . The best synthesis result is the one, having the largest $\Omega_{\text {s }}$ value.

The values of criteria effectiveness of each step of each net program branch are summed, when priorities are determined using the same coefficients of effectiveness. The weights are summed using formula 6 .

It is possible to calculate the priorities of alternatives using the ideal point method.

The methods mentioned above were used to solve real life construction problems.

Leonas USTINOVIČIUS. Doctor, Associate Professor. Dept of Construction Technology and Management. Vilnius Gediminas Technical University (VGTU), Sauletekio al. 11, 2040 Vilnius, Lithuania.

A graduate of Vilnius Civil Engineering Institute (now VGTU) (1982). Doctor (1989). Author of 69 articles. Research interests: building technology. 